\title{
A comparison of the efficacy and safety of morphine and pethidine as analgesia for suspected renal colic in the emergency setting
}

\author{
Alan O’Connor, Stephan A Schug, Hester Cardwell
}

\begin{abstract}
Objectives-The aim of this study was to compare morphine and pethidine in patients with clinically suspected renal colic with regard to analgesic efficacy, patient satisfaction and side effects.

Methods-Double blinded, randomised controlled trial.

Results-There was no significant difference between morphine and pethidine with respect to any of the parameters measured.

Conclusion-Because of the well known adverse effects that may be associated with pethidine use, the authors recommend that morphine should be the preferred agent in suspected renal colic, when an opioid analgesic is to be used. (f Accid Emerg Med 2000;17:261-264)
\end{abstract}

Keywords: renal colic; pethidine; morphine

Renal colic is an important and frequent occurrence in medical practice. It affects $1-5 \%$ of the population in industrialised countries, with a lifetime risk of $20 \%$ in white men and $5-10 \%$ in women. ${ }^{1}$ Typically caused by obstruction of the ureter by a calculus, it is one of the most severe pains experienced by humans. The main goal of the emergency department is the relief of this pain until spontaneous passage of the calculus occurs, or until surgical management is started. A variety of drugs is used for this condition, including antispasmodics, non-steroidal anti-inflammatory agents (NSAIDs) and opioids. ${ }^{2}$

The choice of analgesia used in the management of acute renal colic is changing, with increasing use of NSAIDs. Most studies have shown these drugs to be as effective as opioids $^{3-7}$ but these trials often use opioids as rescue medications. One study in Australia did not show NSAIDs to reduce the need for opioids. ${ }^{8}$ Opioids are still the most frequently prescribed analgesic for renal colic in Australia and pethidine is the most widely used for this condition. ${ }^{9}$ Pethidine has traditionally been used for renal colic, as it is believed to be less likely than morphine to cause ureteric spasm. ${ }^{10}$ However, there is conflicting evidence of the effects of opioids on the ureter. Pethidine has been shown to cause an initial spasmogenic effect followed by a decline and subsequent cessation in ureteric contractility. ${ }^{10}$ Morphine has variously been described as increasing the motility of the ureter, ${ }^{11}$ of having no effect ${ }^{12}{ }^{13}$ and of decreasing ureteric peristalsis. ${ }^{14}$
There are, however, no studies comparing the efficacy of the two drugs in humans, in the clinical setting of renal colic. The purpose of this study was to compare the efficacy, safety, and patient satisfaction of morphine with pethidine, as analgesia for renal colic in the emergency setting.

\section{Methods}

The study was carried out in the emergency department of Auckland Hospital, an urban, tertiary care teaching hospital with 560 beds. It was conducted in accordance with the provisions of the Declaration of Helsinki. Ethics committee approval was gained before the start of the study.

\section{PATIENTS}

Patients 18 to 65 years old who presented to the emergency department with symptoms and signs suggestive of renal colic were considered for enrolment in the trial. Exclusion criteria included a history of allergy to morphine, pethidine or any opioid, a history of opioid abuse, known hepatic or renal disease, and an inability or unwillingness to give verbal and written consent.

Patients were recruited by their primary nurse or doctor when suspected renal colic was first diagnosed and opioid analgesia was felt to be appropriate by the prescribing doctor.

The diagnosis of renal colic was made on clinical grounds by a consultant emergency physician, a specialist registrar, or a senior house officer after consultation. In the interests of prompt analgesia for the patients involved, this diagnosis was made before radiological imaging or haematological investigations, but after urine analysis. All patients did have unilateral abdominal pain positive urine analysis for haematuria, but no formal criteria were laid down for the diagnosis of renal colic in the study procedure. This is because senior clinicians were assessing the patients, and the trial proposition was for patients with clinically suspected rather than verified renal colic.

The trial was explained to the patient and those that gave verbal consent were included. It was felt that, as these patients were in acute pain that verbal consent alone was adequate before starting the study. This was approved by the ethics committee. At the conclusion of the trial, the patient was given a trial sheet explaining the trial and asked to give written consent to inclusion as a confirmation of their previous verbal consent. Only those patients who signed this consent form were to be included in our 
Adult iv opioid protocol

For use in clinical trial comparing pethidine and morphine for analgesia in renal colic

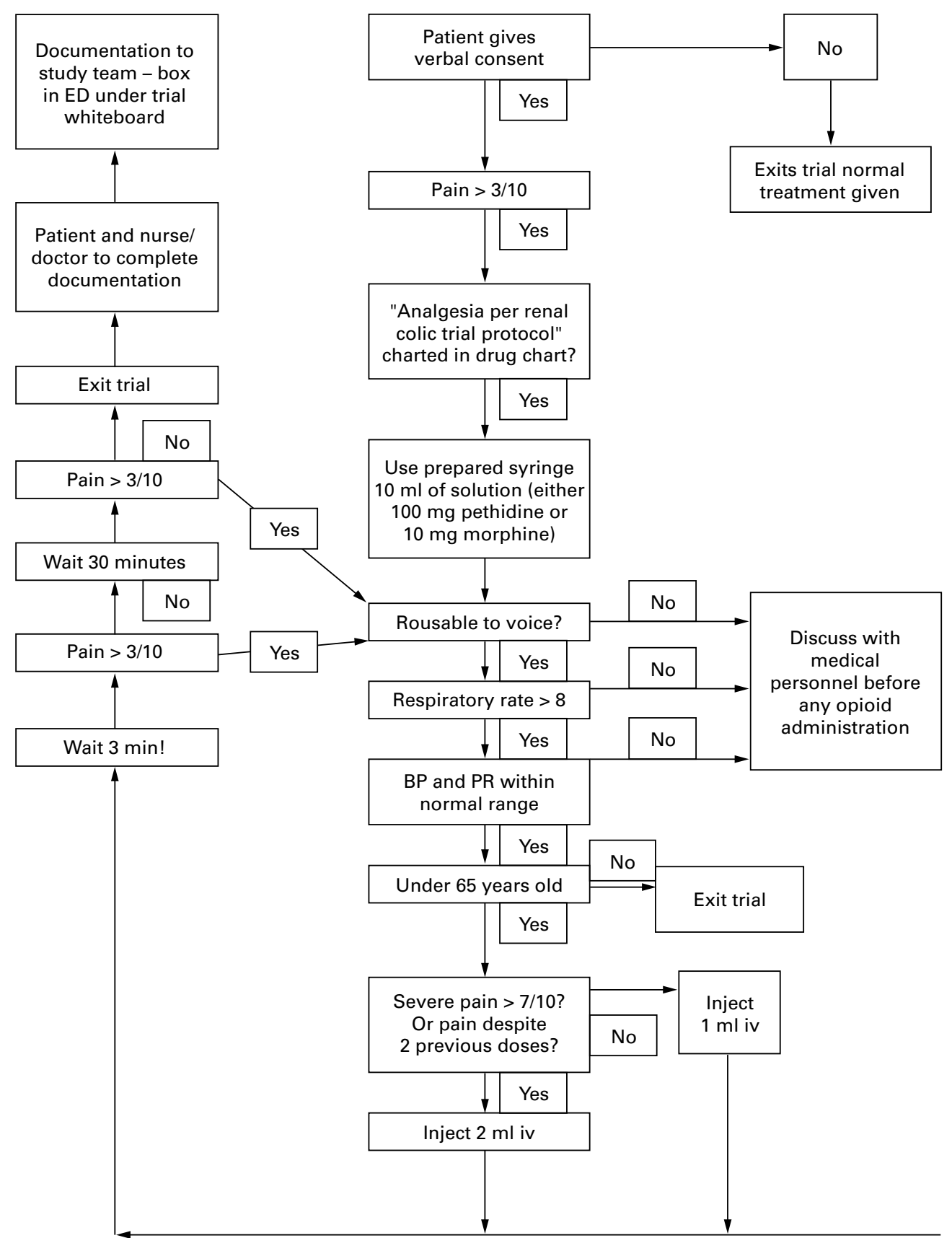

Figure 1 Trial flow sheet for intravenous opioid administration.

analysis-however, no patient refused to give written consent at this stage.

STUDY PROCEDURE

A trial syringe was allocated to each patient using a computer generated randomisation list. Each syringe contained $10 \mathrm{ml}$ of solution, either $10 \mathrm{mg}$ of morphine or $100 \mathrm{mg}$ of pethidine. The patient was asked to plot their pain score on a visual analogue scale (VAS). The VAS was a $100 \mathrm{~mm}$, unnumbered horizontal scale bounded by the descriptors "no pain" and "worst pain imaginable". Each patient was then given $10 \mathrm{mg}$ of intravenous metoclopramide as antiemetic prophylaxis, before administration of analgesia.

Analgesia was titrated as per the trial flow sheet (fig 1) according to the current hospital protocol for opioid analgesia, which was adapted for use in this trial. The patient's pain score was recorded 30 minutes after the start of the trial.

The trial finished when either the patient had a pain score of less than 3 for 30 minutes after the last analgesic dose, or the $10 \mathrm{ml}$ of solution in the syringe was used up. On 
completion of the trial protocol, the patient's final pain score was recorded, and their satisfaction score was charted using a VAS. The patient then exited the trial and received standard analgesia as required. Any adverse effects were noted during treatment and on completion of the trial.

STATISTICAL METHODS

A sample of 45 patients in each treatment group would give a power of $80 \%$ at the 0.05 level of significance to detect a difference of 1.5 in mean pain scores, 30 minutes after the drugs are first administered. Ordinal logistic regression was used to compare the satisfaction VAS and the amount of drug given. A generalised linear model was used to investigate the differences in pain score between the two groups. All efficacy data were analysed on an "intention to treat basis".

\section{Results}

PATIENT RECRUITMENT

One hundred and three non-consecutive patients whose initial diagnosis in the emergency department was acute renal colic were recruited to the trial. The study period was over 18 months. Nine of the 103 patients were withdrawn from the study, four from the pethidine group and five from the morphine group. Five of those patients withdrawn were over 65 years of age (inclusion criteria, 18 to 65 years old), three had inadequate or incomplete trial documentation and one patient was given two syringes of trial solution. Thus, 94 patients were eventually included in the analysis. Fifty four patients were assigned to the pethidine group and 40 patients to the morphine group. The average age of patients in the pethidine group was 43 years and in the morphine group, 38 years. There were more women in the pethidine group $(n=16)$ than in the morphine group $(\mathrm{n}=1)$.

PAIN SCORES

The pethidine group had a median initial pain score of 8.00 and the morphine group a median pain score of 8.05. A generalised linear model including initial pain score, age and sex of the patient and whether or not they were sedated or felt nauseous, found no difference in the pain score after 30 minutes between the two groups of patients $(F 1,87=1.13 ; p=0.3)$. The median pain scores at 30 minutes were pethidine 3.25 , and morphine 3.80 .

The amount of drug needed, up to a maximum of $10 \mathrm{ml}$, to ensure a pain score of less than three, for 30 minutes was used as a measure of final outcome. A generalised linear model with initial pain score, age and sex of the

Table 1 Calculated medians

\begin{tabular}{llll}
\hline & $\begin{array}{l}\text { Morphine } \\
\text { median } \\
\text { values }\end{array}$ & $\begin{array}{l}\text { Pethidine } \\
\text { median } \\
\text { values }\end{array}$ & p Value \\
\hline Initial pain score (0-10) & 8.05 & 8.00 & \\
Pain score at 30 minutes & 3.80 & 3.25 & 0.3 \\
Pain score at end of study & 1.50 & 1.85 & 0.3 \\
Satisfaction score (0-10) & 9.10 & 8.55 & 0.9 \\
Amount of drug given (ml) & 8.00 & 8.00 & \\
\hline
\end{tabular}

Table 2 Number of patients experiencing adverse effects

\begin{tabular}{lll}
\hline & Morphine & Pethidine \\
\hline Nausea and/or vomiting & 4 & 7 \\
Sedation & 3 & 3 \\
Respiratory depression & Nil & Nil \\
Other & Nil & Nil \\
\hline
\end{tabular}

patient included as explanatory variables found no difference between the two drugs in the amount of drug given $(\mathrm{F} 1,89=1.75 ; \mathrm{p}=0.2)$.

Table 1 shows the median values.

PATIENT SATISFACTION

An ordinal logistic regression was used to determine whether the drug used had any effect on the patient's satisfaction score. Included in the analysis were the age and sex of the patient, initial pain score, amount of drug given and whether or not they were sedated or felt nauseous. No difference in satisfaction score was found between the two groups $(\chi 1 \mathrm{df}=2.79 ; \mathrm{p}=0.09)$. The median satisfaction scores were 8.55 for pethidine and 9.10 for the morphine group. The amount of drug given was found to have an effect on the satisfaction score, with those patients who were given the most drug the least satisfied $\left(\chi^{2} 1 \mathrm{df}=\right.$ $4.96 ; \mathrm{p}=0.02)$.

SIDE EFFECTS

The side effects were recorded under four headings and the results are shown in table 2. There was no significant difference between the two groups in the occurrence of side effects. The $\chi^{2}$ test with Fisher's exact test, for nausea and vomiting, gave a $\mathrm{p}$ value of 0.75 .

\section{Discussion}

Although opioids are commonly used as analgesics for patients with acute renal colic, there are few studies comparing different agents. Pethidine has traditionally been used despite its many disadvantages in the clinical setting.

Pethidine is a synthetic opioid, which is about one tenth as potent as morphine. Published studies on the equivalent doses of intravenous morphine and pethidine give an equivalence ratio of $1: 7.5$ to $1: 12.5 .^{15} 16 \mathrm{We}$ used a ratio of $1: 10$ in this trial.

Pethidine is more lipid soluble than morphine and has an elimination half life of two to four hours and duration of action of only two to three hours. Its increased lipid solubility means a more rapid onset of central nervous system effects that increases its abuse potential and makes it more attractive to drug seekers.

The predominant metabolite, norpethidine, is potentially toxic, causing central nervous system excitability such as tremors, myoclonus or seizures. Norpethidine is excreted in the urine and has a terminal half life of 15 to 20 hours, which can increase in renal failure to as long as 40 hours. ${ }^{17}$ Accumulation of norpethidine has also been seen in patients with normal kidneys, particularly with large doses or frequent dosing intervals. ${ }^{18}{ }^{19}$ Naloxone does not reverse pethidine induced seizures. Severe drug interactions may occur with monoamine 
oxidase inhibitors, which can precipitate central nervous system excitation or depression. Pethidine is not recommended for use in the elderly, particularly those with impaired renal function. ${ }^{20}$

With pethidine, the lack of analgesic potency, its short duration of action, abuse potential and adverse effects with repeated doses, means that there is little to recommend this drug.

The usual opioid side effect profiles of morphine and pethidine are similar, with respiratory depression, cough suppression, nausea and vomiting, urinary retention and constipation the most frequent. ${ }^{21}$

We hypothesised that, of the two drugs, morphine is at least as efficacious as pethidine with equivalent patient satisfaction and that it should be the opioid of choice for acute renal colic, as it does not have the potential for the problems that are of concern with pethidine.

Our study suggests that there is no difference in the efficacy of analgesia, and side effect profile between morphine and pethidine. There was no difference in patient satisfaction between the two groups, but those patients who had higher pain scores, also had higher requirements for analgesia and were less satisfied.

Thus, morphine can be used in this condition with no disadvantage to the patient as regards pain relief and escaping the potential complications of pethidine. Metoclopramide has been shown to have an analgesic effect in ureteric colic, ${ }^{22}$ but as it was given in both studies groups, it had no effect on the trial results.

Our study was based on the "intention to treat" and thus included all patients, except for the exclusion criteria as above, in whom the initial diagnosis on presentation, by the attending physician, was renal colic. Thus, confirmation of the diagnosis was not required for inclusion in the study. This more accurately reflects the emergency clinical setting where analgesia is required before diagnostic tests are completed.

However, a separate analysis was performed, excluding those patients in whom a discharge diagnosis different from that of renal colic was eventually made. There was no notable difference in the results.

While we recognise that NSAIDs are becoming more widely used in acute renal colic, there is often a need for supplemental opioid analgesia. Where no contraindication exists, we recommend the use of morphine rather than pethidine if opioid analgesia is required in suspected renal colic.

\section{Contributors}

Alan O'Connor initiated the study, designed the protocol and supervised the trial in the emergency department. He also collected the data and wrote the paper. Stephen Schug aided preparation of the study and ethics committee application, was the main pharmaceutical advisor and edited the final paper. He also acts as guarantor for the paper. Hester Cardwell was involved in the statistical analysis and also in editing the final manuscript. Elizabeth Robinson (Auckland University) aided with the statistical analysis. Amanda Lockney (Pharmacy Manager, Auckland hospital) and Mr. Mark Taylor, aided with coordination of pharmaceutical supplies for the trial, was largely responsible for smooth running of the trial.

Funding: Research Support Fund, Section of Anaesthetics, University of Auckland.

Conflicts of interest: none.

1 Balaji KC, Menon M. Mechanism of stone formation. Urol Clin North Am 1997;24:1-11.

2 Labrecque M, Dostaler LP, Rousselle R, et al. Efficacy of nonsteroidal anti-inflammatory drugs in the treatment of acute renal colic. A meta-analysis [see comments]. Arch acute renal colic. A meta-an

3 Intern Med 1994;154:1381-7. intravenous ketorolac, meperidine, and both (balanced
analgesia) for renal colic [see comments]. Ann Emerg Med analgesia) for re

4 Flannigan GM, Clifford RP, Carver RA, et al. Indomethacin - an alternative to pethidine in ureteric colic. Brf Urol 1983;55:6-9.

5 Hetherington JW, Philp NH. Diclofenac sodium versus pethidine in acute renal colic. BMF Clin Res Ed. 1986;292: $237-8$.

6 Sjodin JG, Holmlund D. Indomethacin by intravenous infusion in ureteral colic. A multicentre study. Scand F Urol Nephrol 1982;16:221-5.

7 Thompson JF, Pike JM, Chumas PD, et al. Rectal diclofenac compared with pethidine injection in acute renal colic. BMF 1989;299:1140-1.

8 Ginifer C, Kelly AM. Administration of rectal indomethacin does not reduce the requirement for intravenous narcotic analgesia in acute renal colic. Eur f Emerg Med 1996;3:92-

9 Morgan T. Renal colic. Austr Fam Physician 1984;13:39901.

10 Lennon GM, Bourke J, Ryan PC, et al. Pharmacological options for the treatment of acute ureteric colic. An in vitro experimental study. Br F Urol 1993;71:401-7.

11 Ross JA, Griffiths JM. Morphine a spasmolytic? BMF 1970; 3:107.

12 Andersson KE, Ulmsten U. Effects of spinal anaesthesia, lidocaine, and morphine, on the motility of the human ureter in vivo. Scand F Urol Nephrol 1975;9:236-42.

13 Struthers NW. An experimental model for evaluating drug effects on the ureter. Br F Urol 1973;45:23-7.

14 Ancill RJ, Jackson DM, Redfern PH. The pharmacology of the rat ureter in vivo. Br $\mathcal{F}$ Pharmacol 1972;44:628-33.

15 Allen T, Blais R. Opioids in emergency medicine, concepts and Allen T, Blais R. Opioids in emergency medicine,

16 Foley KM. The treatment of cancer pain. $N$ Engl f Med 1985;313:84-95.

17 Szeto HH, Inturrisi CE, Houde R, et al. Accumulation of norpethidine, an active metabolite of meperidine, in patients with renal failure of cancer. Ann Intern Med 1977; 86:738-41.

18 Marinella MA. Meperidine-induced generalized seizures with normal renal function. South Med F 1997;90:556-8.

19 Mauro VF, Bonfiglio MF, Spunt AL. Meperidine-induced seizure in a patient without renal dysfunction or sickle cell anemia. Clin Pharmacol 1986;5:837-9.

20 Forman WB. Opioid analgesic drugs in the elderly. Clin Geriatr Med 1996;12:489-500.

21 Duthie DJ, Nimmo WS. Adverse effects of opioid analgesic drugs. Br F Anaesth 1987;59:61-77.

22 Hedenbro JL, Olsson AM. Metoclopramide and ureteric colic. Acta Chir Scand 1988;154:439-40. 